The valence density of urea at $123 \mathrm{~K}$ has been reported in a previous paper by Mullen \& Hellner (1978). A comparison with the thiourea molecules described here shows that the peak height at $\mathrm{N}$ in urea (3.75 e $\left.\AA^{-3}\right)$ is very close to the values found for both thiourea molecules $\left(3.62\right.$ and $\left.3.69 \mathrm{e}^{-3}\right)$. In contrast, the peak height at $\mathrm{O}$ in urea $\left(7.0 \mathrm{e} \AA^{-3}\right)$ is considerably higher than those at $\mathrm{S}$ in thiourea. The mean $\mathrm{C}-\mathrm{S}$ bond population (3.03 e) may be compared with a value of 3.27 e for $\mathrm{C}-\mathrm{O}$ in urea.

The $\mathrm{C}-\mathrm{N}$ bond populations (e) compare very closely: 1.89 in urea; 1.85 and 1.92 in thiourea (Table 2). The $\mathrm{N}-\mathrm{H}$ bond populations are lower in thiourea $(1.15 \mathrm{e})$ than in urea $(1.33 \mathrm{e})$, mean values being quoted in both cases. The populations of the residual charge at the protons show the opposite trend with mean values of 0.67 and 0.39 e for thiourea and urea respectively.

Lone-pair populations at $\mathrm{S}$ in thiourea are much lower (mean $=2.88 \mathrm{e}$ ) than at $\mathrm{O}$ in urea $(4.16 \mathrm{e})$. The $\mathrm{N}$ lone pair also has a lower population in thiourea $(1.64 \mathrm{e})$ than in urea $(2.36 \mathrm{e})$.

It is intended in future papers in this series to publish experimental deformation densities and to compare these with quantum chemically derived densities. Additionally, form factors for bond charges obtained from the experimental model (Scheringer, Kutoglu, Mullen \& Hellner, 1978) will be compared with those of Fritchie (1966) and Cromer \& Larson (1974).

\section{References}

Cromer, D. T. \& Larson, A. C. (1974). J. Chem. Phys. 60, $176-184$.

Dvoryankina, V. F. \& Vainshtein, B. K. (1960). Kristallografiya, 5, 589-599.

Elcombe, M. M. \& Taylor, J. C. (1968). Acta Cryst. A24, $410-420$.

FINGER, L. (1972). A Fortran IV computer program for structure factor calculation and least-squares refinement of crystal structures. Carnegie Institution of Washington.

Fritchie, C. J. (1966). Acta Cryst. 20, 27-36.

Goldsmith, G. J. \& White, J. G. (1959). J. Chem. Phys. 31, 1175-1187.

Hamilton, W. C. (1965). Acta Cryst. 18, 502-510.

Hellner, E. (1977). Acta Cryst. B33, 3813-3816.

International Tables for X-ray Crystallography (1952). Vol. I. Birmingham: Kynoch Press.

International Tables for X-ray Crystallography (1974). Vol. IV. Birmingham: Kynoch Press.

JoHnson, C. K. (1965). ORTEP. Report ORNL-3794. Oak Ridge National Laboratory, Tennessee.

Mullen, D. \& Hellner, E. (1977). Acta Cryst. B33, 38163823.

Mullen, D. \& Hellner, E. (1978). Acta Cryst. B34, 16241627.

ReEs, B. (1976). Acta Cryst. A32, 483-488.

Scheringer, C., Kutoglu, A., Mullen, D. \& Hellner, E. (1978). Acta Cryst. A34, 475-476.

Stewart, R. F. (1976). Acta Cryst. A 32. 565-574.

Truter, M. R. (1967). Acta Cryst. 22, 556-559.

Acta Cryst. (1978). B34, 2794-2798

\title{
The Crystal and Molecular Structure of $2^{\prime}, 3^{\prime}, 5^{\prime}$-Tri- $O$-acetyl-6- $O$-(mesitylenesulphonyl)guanosine
}

\author{
By S. NeIDLE AND A. ACHARI \\ Department of Biophysics, University of London, King's College, 26-29 Drury Lane, London WC2B 5RL, \\ England
}

G. M. SHELDRICK

University Chemical Laboratory, Lensfield Road, Cambridge CB2 1 EW, England

AND C. B. ReEse AND P. K. BRIDSON

Department of Chemistry, University of London, King's College, Strand, London WC2R $2 L S$, England

(Received 22 March 1978; accepted 21 April 1978)

\begin{abstract}
The structure of a 6-O-mesitylenesulphonyl derivative of $2^{\prime}, 3^{\prime}, 5^{\prime}$-tri- $O$-acetylguanosine, $\mathrm{C}_{24} \mathrm{H}_{29} \mathrm{~N}_{5} \mathrm{O}_{10} \mathrm{~S}$, has been determined by X-ray diffraction. Crystals are monoclinic, $a=26 \cdot 370(4), b=8 \cdot 200(2), c=$ 17.991 (3) $\AA, \beta=132.77$ (4) ${ }^{\circ}$. The solution of the structure in space group $C 2$ was not straightforward and is described in detail. Refinement converged at $R=0.110$ for 1102 observed reflections. The guanine base displays some deviations from its usual geometry due to the loss of $\mathrm{C}(6)-\mathrm{O}(6)$ double-bond character. The ribose sugar is $\mathrm{C}\left(2^{\prime}\right)$-endo puckered.
\end{abstract}




\section{Introduction}

The title compound (I) was obtained by treatment of guanosine (fully protected by acetyl groups on the sugar hydroxyl positions), with mesitylenesulphonyl chloride (Bridson, Markiewicz \& Reese, 1977). The proposed structure, with the $O(6)$ atom of guanine rather than $N(2)$ being the site of attack, was verified by chemical and spectroscopic means. The crystallographic analysis reported here provides definitive confirmation for this assignment. The $\mathrm{O}(6)$ oxygen is a favoured site for the binding of various mutagenic agents to guanine in nucleic acids.

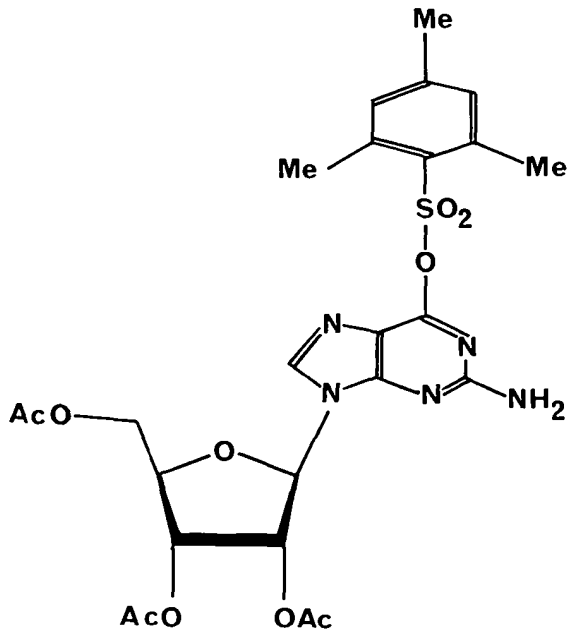

(I)

\section{Experimental}

The title compound was crystallized from aqueous solution as elongated, extremely thin fibrous platelets. These showed parallel extinction under the polarizing microscope. Photographs revealed the crystals to be monoclinic. Systematic absences $h k l: h+k=2 n+1$ and $0 k 0: k=2 n+1$, together with the known optical activity of the compound, uniquely identified the space group as $C 2$. Cell dimensions were obtained from diffractometer measurements.

\section{Crystal data}

$\mathrm{C}_{24} \mathrm{H}_{29} \mathrm{~N}_{5} \mathrm{O}_{10} \mathrm{~S}, M_{r}=579.0$, monoclinic plates, elongated along $b . a=26.370$ (4), $b=8.200(2), c=$ 17.991 (3) $\AA, \beta=132.77(4)^{\circ}, V=2855.8 \AA^{3}, D_{m}=$ 1.33 (by flotation), $D_{c}=1.346 \mathrm{~g} \mathrm{~cm}^{-3}$ for $Z=4$. $F(000)=1376, \mu=14.9 \mathrm{~cm}^{-1}$ for $\mathrm{Cu} K \alpha$. radiation $\left(\lambda_{K \alpha}=1.54178 \AA\right)$. Space group $C 2\left(\right.$ No. $\left.5, C_{2}^{3}\right)$.

Intensities were collected on an Enraf-Nonius CAD4 automatic diffractometer, to a $\theta$ limit of $60^{\circ}$ for $\mathrm{Cu} \mathrm{Ka}$ radiation. A total of 1597 reflections were measured, of which only 1102 were considered to be observable, with $F>2 \sigma(F)$.

\section{Structure solution and refinement}

Initial attempts to solve the structure by conventional direct methods, heavy-atom location or search procedures, were all unsuccessful. An unconventional direct-methods approach, detailed below, eventually led to the correct solution.

Table 1. Final positional parameters $\left(\times 10^{4}\right)$ for the non-hydrogen atoms, and isotropic $(U)$ temperature factors $\left(\AA^{2} \times 10^{3}\right)$ with estimated standard deviations in parentheses

$\begin{array}{lcrcr} & x & y & z & U \\ \mathrm{C}(16) & 3959(7) & 6529(16) & 9100(9) & 31(5) \\ \mathrm{C}(26) & 3967(7) & 6090(16) & 8359(9) & 41(5) \\ \mathrm{C}(36) & 4044(7) & 7285(16) & 7890(9) & 48(6) \\ \mathrm{C}(46) & 4112(7) & 8919(16) & 8161(9) & 49(6) \\ \mathrm{C}(56) & 4104(7) & 9358(16) & 8902(9) & 56(6) \\ \mathrm{C}(66) & 4027(7) & 8163(16) & 9371(9) & 42(6) \\ \mathrm{C}(76) & 3891(13) & 4321(30) & 8025(17) & 67(7) \\ \mathrm{C}(86) & 4145(13) & 10238(34) & 7572(17) & 74(7) \\ \mathrm{C}(96) & 4066(12) & 8878(32) & 10157(15) & 63(7) \\ \mathrm{O}\left(1^{\prime}\right) & 2165(6) & -3111(18) & 6795(9) & 39(4) \\ \mathrm{C}\left(1^{\prime}\right) & 2016(9) & -2352(28) & 7309(14) & 40(5) \\ \mathrm{C}\left(2^{\prime}\right) & 1228(8) & -2531(23) & 6633(12) & 20(4) \\ \mathrm{O}\left(2^{\prime}\right) & 1055(6) & -2370(19) & 7232(9) & 44(4) \\ \mathrm{C}(21) & 447(11) & -1834(30) & 6809(17) & 56(6) \\ \mathrm{O}(21) & 7(9) & -1345(26) & 5948(13) & 95(6) \\ \mathrm{C}(22) & 313(12) & -1755(34) & 7496(17) & 70(7) \\ \mathrm{C}\left(3^{\prime}\right) & 1107(9) & -4290(23) & 6203(12) & 28(5) \\ \mathrm{O}\left(3^{\prime}\right) & 1291(6) & -5442(19) & 6946(9) & 44(4) \\ \mathrm{C}(31) & 777(11) & -5957(28) & 6847(15) & 50(6) \\ \mathrm{O}(31) & 166(8) & -5462(22) & 6198(11) & 78(5) \\ \mathrm{C}(32) & 1032(12) & -7162(34) & 7708(17) & 70(7) \\ \mathrm{C}\left(4^{\prime}\right) & 1666(10) & -4391(26) & 6169(13) & 38(5) \\ \mathrm{C}\left(5^{\prime}\right) & 1330(10) & -4086(29) & 5080(14) & 47(6) \\ \mathrm{O}\left(5^{\prime}\right) & 1877(7) & -4013(20) & 5093(9) & 52(4) \\ \mathrm{C}(51) & 2000(9) & -2626(29) & 4857(13) & 42(5) \\ \mathrm{O}(51) & 1616(9) & -1452(27) & 4561(12) & 91(6) \\ \mathrm{C}(52) & 2582(12) & -2726(34) & 4914(19) & 71(7) \\ \mathrm{N}(1) & 2282(7) & 2774(21) & 9516(10) & 29(4) \\ \mathrm{C}(2) & 2676(10) & 1290(27) & 9572(14) & 38(5) \\ \mathrm{N}(2) & 2689(8) & 1140(23) & 10337(11) & 51(5) \\ \mathrm{N}(3) & 2449(7) & 32(20) & 8959(10) & 32(4) \\ \mathrm{C}(4) & 2452(8) & 358(22) & 8245(11) & 21(4) \\ \mathrm{C}(5) & 2637(9) & 1754(24) & 8084(13) & 29(5) \\ \mathrm{C}(6) & 2865(10) & 2972(27) & 8769(15) & 42(6) \\ \mathrm{O}(6) & 3102(6) & 4464(17) & 8760(8) & 38(4) \\ \mathrm{N}(7) & 2533(8) & 1702(23) & 7212(11) & 39(5) \\ \mathrm{C}(8) & 2272(10) & 288(29) & 6848(14) & 40(5) \\ \mathrm{N}(9) & 2210(7) & -636(19) & 7445(10) & 27(4) \\ \mathrm{S} & 3885(3) & 5062 & 9708(4) & * \\ \mathrm{O}(16) & 3883(9) & 5704(23) & 10429(10) & * \\ \mathrm{O}(26) & 4282(8) & 3674(25) & 9964(11) & *\end{array}$

* The anisotropic thermal parameters for these atoms are:

$\begin{array}{lcllclc} & U_{11} & U_{22} & U_{33} & U_{23} & U_{13} & U_{12} \\ \mathrm{~S} & 49(3) & 57(4) & 32(3) & 18(3) & 21(3) & 0(4) \\ \mathrm{O}(16) & 146(16) & 87(13) & 43(9) & -34(9) & 74(11) & -49(12) \\ \mathrm{O}(26) & 59(11) & 98(15) & 76(12) & 35(12) & 37(10) & 1(13)\end{array}$


A large starting set was constructed containing some negative sign information. Four $k=0$ reflections were assigned phase angles $(\varphi)$ of $0^{\circ}$, and four more were assigned $\varphi=180^{\circ}$, all on the basis of $\sum_{1}$ relations and seminvariant relations derived by combining triplet and quartet phase relationships (Sheldrick, 1976). Two trio relationships were added, each of one positive and two negative reflection quartets involving only $k=0$ reflections. The total starting set included $18 k=0$ reflections and one $k=1$ reflection (to fix the origin along $b$ ), plus six general reflections which were assigned the 'magic integer' phases $33 x, 49 x, 57 x, 61 x$, $63 x$ and $64 x$ (Main, 1977). After weighted tangentformula expansion to 250 of the largest $E$ values, the figures of merit NQEST (De Titta, Edmonds, Langs \& Hauptman, 1975) and $R_{\alpha}$ (Roberts, Pettersen, Sheldrick, Isaacs \& Kennard, 1973) were calculated. The 'best' solutions were expanded to $461 E$ values $>1 \cdot 1$. One $E$ map (ranked 25th on NQEST and 2nd on $R_{\alpha}$ ) revealed a fragment $\left(\mathrm{O}_{2} \mathrm{~S}-\mathrm{O}-\right)$ attached to a sixmembered aromatic ring, with a further atom attached ortho to the ring. Tangent expansion (Karle, 1968) was attempted, but did not develop the structure further.

\section{Table 2. Final positional parameters for the hydrogen atoms}

Each hydrogen atom was assigned a temperature factor $(U) 0.005$ $\AA^{2}$ greater than the atom to which it is attached. The numbering scheme follows that of the non-hydrogen atom to which each hydrogen is attached.

\begin{tabular}{lrrr} 
& \multicolumn{1}{c}{$x$} & \multicolumn{1}{c}{$y$} & \multicolumn{1}{c}{$z$} \\
$\mathrm{H}(36)$ & 405 & 694 & 732 \\
$\mathrm{H}(56)$ & 416 & 1062 & 911 \\
$\mathrm{H}(761)$ & 392 & 408 & 746 \\
$\mathrm{H}(762)$ & 340 & 391 & 775 \\
$\mathrm{H}(763)$ & 430 & 368 & 872 \\
$\mathrm{H}(861)$ & 416 & 980 & 702 \\
$\mathrm{H}(862)$ & 462 & 1082 & 818 \\
$\mathrm{H}(863)$ & 372 & 1108 & 721 \\
$\mathrm{H}(961)$ & 412 & 1016 & 1034 \\
$\mathrm{H}(962)$ & 454 & 825 & 1077 \\
$\mathrm{H}(963)$ & 365 & 837 & 1006 \\
$\mathrm{H}\left(1^{\prime}\right)$ & 227 & -294 & 803 \\
$\mathrm{H}\left(2^{\prime}\right)$ & 94 & -161 & 605 \\
$\mathrm{H}(221)$ & -16 & -110 & 712 \\
$\mathrm{H}(222)$ & 73 & -119 & 822 \\
$\mathrm{H}(223)$ & 26 & -300 & 762 \\
$\mathrm{H}\left(3^{\prime}\right)$ & 57 & -443 & 551 \\
$\mathrm{H}(321)$ & 63 & -769 & 765 \\
$\mathrm{H}(322)$ & 136 & -641 & 838 \\
$\mathrm{H}(323)$ & 134 & -812 & 776 \\
$\mathrm{H}\left(4^{\prime}\right)$ & 191 & -557 & 647 \\
$\mathrm{H}\left(5^{\prime} 1\right)$ & 108 & -290 & 483 \\
$\mathrm{H}\left(5^{\prime} 2\right)$ & 95 & -501 & 457 \\
$\mathrm{H}(521)$ & 276 & -154 & 491 \\
$\mathrm{H}(522)$ & 230 & -329 & 418 \\
$\mathrm{H}(523)$ & 302 & -347 & 549 \\
$\mathrm{H}(21)$ & 224 & 125 & 1025 \\
$\mathrm{H}(22)$ & 309 & 45 & 1101 \\
$\mathrm{H}(8)$ & 218 & -48 & 628
\end{tabular}

Since it was suspected that the fragment was correctly oriented but wrongly positioned with respect to the space-group origin, a tangent formula expansion was performed in space group $C 1$, with the data expanded to a full hemisphere. Two iterations revealed two $\left(\mathrm{O}_{2} \mathrm{~S}-\mathrm{O}-\right)$ groups, each attached to an almost complete guanine ring, related by a twofold axis at $x=$ $0 \cdot 178, z=0 \cdot 166$. Coordinates were calculated for space group $C 2$ with the origin shifted to this position, and three cycles of tangent expansion with $E$ values $>0.9$ developed the complete structure.

The structure was refined by full-matrix least-squares techniques. In view of the paucity of observable reflection data, the benzene ring of the mesitylene group was treated as a rigid body, and only the $S$ atoms and two $O$ atoms were refined anisotropically. The positions of all hydrogen atoms were calculated, and their structure-factor contributions taken into account during refinement. The final $R$ was $0 \cdot 110$, and $R_{w}$ was $0 \cdot 100$, where $w=\left[0.8303 / \sigma^{2}\left(F_{o}\right)+0.005 F_{o}^{2}\right]$.

All calculations were performed with programs written by GMS; diagrams were drawn with the aid of the program PLUTO (Motherwell, 1975).

Tables 1 and 2 list the final positional and thermal parameters.*

\section{Discussion}

Interatomic bond lengths and angles are given in Table 3; the numbering scheme used is shown in Fig. 1. Due

* A list of structure factors has been deposited with the British Library Lending Division as Supplementary Publication No. SUP 33575 (6 pp.). Copies may be obtained through The Executive Secretary, International Union of Crystallography, 5 Abbey Square, Chester CH1 2HU, England.

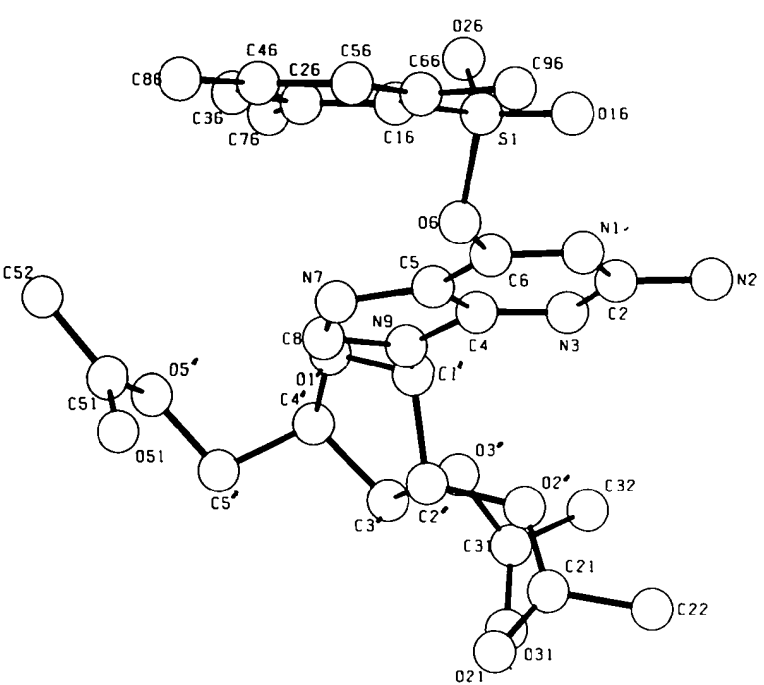

Fig. 1. A view of the molecule showing the numbering scheme used. 
to the relatively poor quality of the crystal, which has resulted in an unusually small observable:variable ratio, the e.s.d.'s are not low.

The analysis has confirmed that $\mathrm{O}(6)$ of the guanine is the site of attachment of the sulphonyl group. The trimethylbenzene group is aligned approximately parallel to the plane of the guanine base (Fig. 1), although, as Fig. 2(a) shows, these two planar groups do not stack on each other at all. The two acetyl moieties at positions $2^{\prime}$ and $3^{\prime}$ on the ribose ring are also aligned roughly parallel to each other, presumably so as to minimize non-bonded repulsions.

The arrangement around the $\mathrm{S}$ atom shows some deviations from the tetrahedral; these are understandable in view of the steric bulk of some of its adjacent groups. Bond lengths involving the $S$ atom are unexceptional within the limits of experimental error. The dimensions of the ribose group are likewise as expected, although there is perhaps an accentuation of the characteristic difference between the $\mathrm{O}\left(1^{\prime}\right)-\mathrm{C}\left(1^{\prime}\right)$ and $\mathrm{O}\left(1^{\prime}\right)-\mathrm{C}\left(4^{\prime}\right)$ bond lengths (Saenger, 1973).

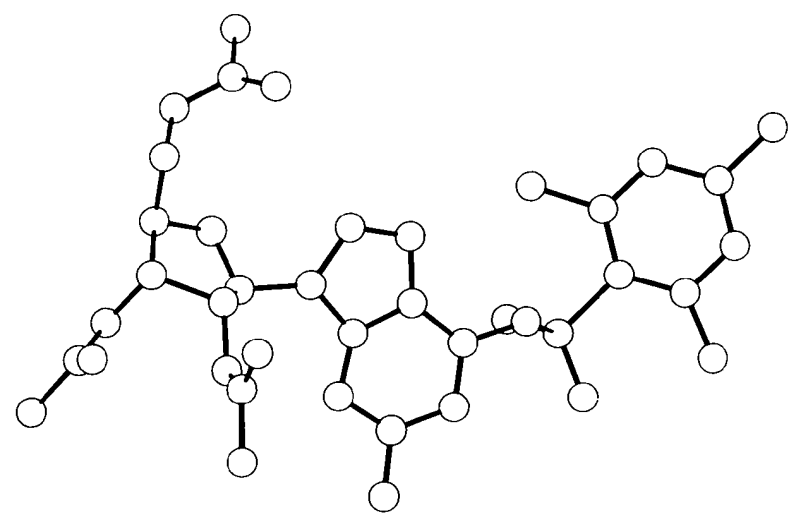

(a)

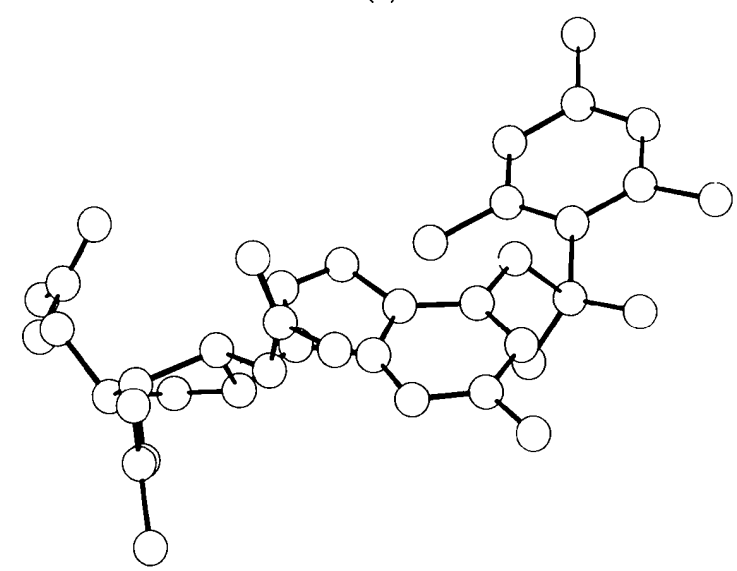

(b)

Fig. 2. (a) A view of the molecule projected onto the guanine plane. (b) A view looking down the $\mathrm{C}\left(4^{\prime}\right)-\mathrm{O}\left(1^{\prime}\right)-\mathrm{C}\left(1^{\prime}\right)$ sugar plane.
The guanine base is flat (Table 4). Its geometry is, however, appreciably different from the base in 9-ethylguanine (Destro, Kistenmacher \& Marsh, 1974) and in guanosine dihydrate (Thewalt, Bugg \& Marsh, 1970), as well as from the averaged values reported by Voet \& Rich (1970). These differences can be ascribed to the $\mathrm{O}(6)$-substitution, which has resulted in a lengthening

\section{Table 3. Bond lengths $(\AA)$ and angles $\left(^{\circ}\right)$}

Estimated standard deviations for bond lengths are between 0.01 and $0.02 \AA$ and 1 and $2^{\circ}$ for bond angles. The bond lengths and angles in the benzene ring of the mesitylene group were constrained to $1.395 \AA$ and $120^{\circ}$ respectively.

$\begin{array}{llll}\mathrm{O}\left(1^{\prime}\right)-\mathrm{C}(1) & 1.37 & \mathrm{~N}(1)-\mathrm{C}(2) & 1.36 \\ \mathrm{O}\left(1^{\prime}\right)-\mathrm{C}(4) & 1.45 & \mathrm{~N}(1)-\mathrm{C}(6) & 1.32 \\ \mathrm{C}\left(1^{\prime}\right)-\mathrm{C}\left(2^{\prime}\right) & 1.55 & \mathrm{C}(2)-\mathrm{N}(2) & 1.36 \\ \mathrm{C}\left(1^{\prime}\right)-\mathrm{N}(9) & 1.46 & \mathrm{C}(2)-\mathrm{N}(3) & 1.32 \\ \mathrm{C}\left(2^{\prime}\right)-\mathrm{O}\left(2^{\prime}\right) & 1.43 & \mathrm{~N}(3)-\mathrm{C}(4) & 1.32 \\ \mathrm{C}\left(2^{\prime}\right)-\mathrm{C}\left(3^{\prime}\right) & 1.56 & \mathrm{C}(4)-\mathrm{C}(5) & 1.35 \\ \mathrm{O}\left(2^{\prime}\right)-\mathrm{C}(21) & 1.30 & \mathrm{C}(4)-\mathrm{C}(9) & 1.38 \\ \mathrm{C}(21)-\mathrm{O}(21) & 1.21 & \mathrm{C}(5)-\mathrm{C}(6) & 1.37 \\ \mathrm{C}(21)-\mathrm{C}(22) & 1.50 & \mathrm{C}(5)-\mathrm{N}(7) & 1.40 \\ \mathrm{C}\left(3^{\prime}\right)-\mathrm{O}\left(3^{\prime}\right) & 1.43 & \mathrm{C}(6)-\mathrm{O}(6) & 1.38 \\ \mathrm{C}\left(3^{\prime}\right)-\mathrm{C}\left(4^{\prime}\right) & 1.52 & \mathrm{~N}(7)-\mathrm{C}(8) & 1.28 \\ \mathrm{O}\left(3^{\prime}\right)-\mathrm{C}(31) & 1.31 & \mathrm{C}(8)-\mathrm{N}(9) & 1.41 \\ \mathrm{C}(31)-\mathrm{O}(31) & 1.25 & \mathrm{O}(6)-\mathrm{S} & 1.62 \\ \mathrm{C}(31)-\mathrm{C}(32) & 1.55 & \mathrm{~S}-\mathrm{O}(16) & 1.40 \\ \mathrm{C}\left(4^{\prime}\right)-\mathrm{C}\left(5^{\prime}\right) & 1.53 & \mathrm{~S}-\mathrm{O}(26) & 1.40 \\ \mathrm{C}\left(5^{\prime}\right)-\mathrm{O}(5) & 1.43 & \mathrm{~S}-\mathrm{C}(16) & 1.72 \\ \mathrm{O}\left(5^{\prime}\right)-\mathrm{C}(51) & 1.33 & \mathrm{C}(26)-\mathrm{C}(76) & 1.53 \\ \mathrm{C}(51)-\mathrm{O}(51) & 1.23 & \mathrm{C}(46)-\mathrm{C}(86) & 1.56 \\ \mathrm{C}(51)-\mathrm{C}(52) & 1.47 & \mathrm{C}(66)-\mathrm{C}(96) & 1.47\end{array}$

$\mathrm{C}\left(1^{\prime}\right)-\mathrm{O}\left(1^{\prime}\right)-\mathrm{C}\left(4^{\prime}\right) \quad 109$

$\mathrm{O}\left(1^{\prime}\right)-\mathrm{C}\left(1^{\prime}\right)-\mathrm{C}\left(2^{\prime}\right) \quad 106$

$\mathrm{O}\left(1^{\prime}\right)-\mathrm{C}\left(1^{\prime}\right)-\mathrm{N}(9) \quad 109$

$\mathrm{C}\left(2^{\prime}\right)-\mathrm{C}\left(1^{\prime}\right)-\mathrm{N}(9) \quad 111$

$\mathrm{C}\left(1^{\prime}\right)-\mathrm{C}\left(2^{\prime}\right)-\mathrm{C}\left(3^{\prime}\right) \quad 101$

$\mathrm{C}\left(1^{\prime}\right)-\mathrm{C}\left(2^{\prime}\right)-\mathrm{O}\left(2^{\prime}\right) \quad 110$

$\mathrm{C}\left(3^{\prime}\right)-\mathrm{C}\left(2^{\prime}\right)-\mathrm{O}\left(2^{\prime}\right) \quad 113$

$\mathrm{C}\left(2^{\prime}\right)-\mathrm{O}\left(2^{\prime}\right)-\mathrm{C}(21) \quad 119$

$\mathrm{O}\left(2^{\prime}\right)-\mathrm{C}(21)-\mathrm{O}(21) \quad 126$

$\mathrm{O}\left(2^{\prime}\right)-\mathrm{C}(21)-\mathrm{C}(22) \quad 115$

$\mathrm{O}(21)-\mathrm{C}(21)-\mathrm{C}(22) \quad 119$

$\mathrm{C}\left(2^{\prime}\right)-\mathrm{C}\left(3^{\prime}\right)-\mathrm{C}\left(4^{\prime}\right) \quad 101$

$\mathrm{C}\left(2^{\prime}\right)-\mathrm{C}\left(3^{\prime}\right)-\mathrm{O}\left(3^{\prime}\right) \quad 109$

$\mathrm{C}\left(4^{\prime}\right)-\mathrm{C}\left(3^{\prime}\right)-\mathrm{O}\left(3^{\prime}\right) \quad 106$

$\mathrm{C}\left(3^{\prime}\right)-\mathrm{O}\left(3^{\prime}\right)-\mathrm{C}(31) \quad 115$

$\mathrm{O}\left(3^{\prime}\right)-\mathrm{C}(31)-\mathrm{O}(31) \quad 125$

$\mathrm{O}\left(3^{\prime}\right)-\mathrm{C}(31)-\mathrm{C}(32) \quad 111$

$\mathrm{O}(31)-\mathrm{C}(31)-\mathrm{C}(32) \quad 123$

$\mathrm{C}\left(3^{\prime}\right)-\mathrm{C}\left(4^{\prime}\right)-\mathrm{O}\left(1^{\prime}\right) \quad 109$

$\mathrm{C}\left(3^{\prime}\right)-\mathrm{C}\left(4^{\prime}\right)-\mathrm{C}\left(5^{\prime}\right) \quad 108$

$\mathrm{C}\left(5^{\prime}\right)-\mathrm{C}\left(4^{\prime}\right)-\mathrm{O}\left(1^{\prime}\right) \quad 108$

$\mathrm{C}\left(4^{\prime}\right)-\mathrm{C}\left(5^{\prime}\right)-\mathrm{O}\left(5^{\prime}\right) \quad 107$

$\mathrm{C}\left(5^{\prime}\right)-\mathrm{O}\left(5^{\prime}\right)-\mathrm{C}(51) \quad 120$

$\mathrm{O}\left(5^{\prime}\right)-\mathrm{C}(51)-\mathrm{O}(51) \quad 120$

$\mathrm{O}\left(5^{\prime}\right)-\mathrm{C}(51)-\mathrm{C}(52) \quad 114$

$\mathrm{O}(51)-\mathrm{C}(51)-\mathrm{C}(52) \quad 126$

$\mathrm{C}(2)-\mathrm{N}(1)-\mathrm{C}(6)$

$\mathrm{N}(1)-\mathrm{C}(2)-\mathrm{N}(2) \quad 115$

$\mathrm{N}(1)-\mathrm{C}(2)-\mathrm{N}(3) \quad 128$

$\mathrm{N}(2)-\mathrm{C}(2)-\mathrm{N}(3)$

$\mathrm{C}(2)-\mathrm{N}(3)-\mathrm{C}(4) \quad 111$

$\mathrm{N}(3)-\mathrm{C}(4)-\mathrm{C}(5) \quad 129$

$\mathrm{N}(3)-\mathrm{C}(4)-\mathrm{N}(9) \quad 126$

$\mathrm{C}(5)-\mathrm{C}(4)-\mathrm{N}(9) \quad 105$

$\mathrm{C}(4)-\mathrm{C}(5)-\mathrm{C}(6) \quad 115$

$\mathrm{C}(4)-\mathrm{C}(5)-\mathrm{N}(7) \quad 113$

$\mathrm{C}(6)-\mathrm{C}(5)-\mathrm{N}(7) \quad 132$

$\mathrm{N}(1)-\mathrm{C}(6)-\mathrm{C}(5) \quad 121$

$\mathrm{N}(1)-\mathrm{C}(6)-\mathrm{O}(6)$

$\mathrm{C}(5)-\mathrm{C}(6)-\mathrm{O}(6) \quad 124$

$\mathrm{S}-\mathrm{O}(6)-\mathrm{C}(6)$

$\mathrm{C}(5)-\mathrm{N}(7)-\mathrm{C}(8) \quad 103$

$\mathrm{N}(7)-\mathrm{C}(8)-\mathrm{N}(9) \quad 113$

$\mathrm{C}(4)-\mathrm{N}(9)-\mathrm{C}(8) \quad 106$

$\mathrm{C}(4)-\mathrm{N}(9)-\mathrm{C}\left(1^{\prime}\right) \quad 127$

$\mathrm{C}(8)-\mathrm{N}(9)-\mathrm{C}\left(\mathrm{I}^{\prime}\right) \quad 127$

O(6)-S-C (16) 99

O(6)-S-O(16) 107

$\mathrm{O}(6)-\mathrm{S}-\mathrm{O}(26)$

$\mathrm{O}(16)-\mathrm{S}-\mathrm{C}(16)$

$\mathrm{O}(16)-\mathrm{S}-\mathrm{O}(26)$

$\mathrm{O}(26)-\mathrm{S}-\mathrm{C}(16)$

$\mathrm{C}(16)-\mathrm{C}(26)-\mathrm{C}(76) \quad 122$

$\mathrm{C}(36)-\mathrm{C}(26)-\mathrm{C}(76) \quad 118$

$\mathrm{C}(36)-\mathrm{C}(46)-\mathrm{C}(86) \quad 119$

$\mathrm{C}(56)-\mathrm{C}(46)-\mathrm{C}(86) \quad 121$

C(16)-C $(66)-C(96) \quad 129$

$\mathrm{C}(56)-\mathrm{C}(66)-\mathrm{C}(96) \quad 111$ 

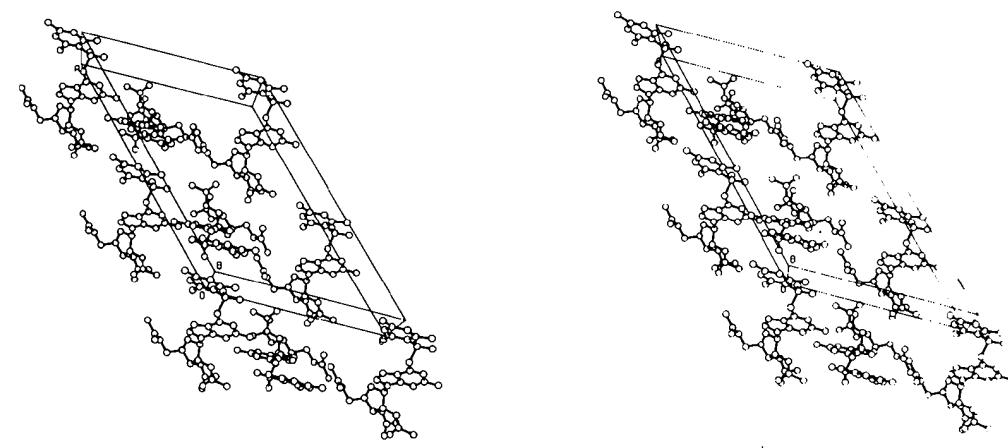

Fig. 3. A stereoview of the packing of molecules in the unit cell.

Table 4. Deviations $(\AA)$ of atoms from various leastsquares planes

An asterisk denotes atoms not included in the plane calculations.

\begin{tabular}{cr}
\multicolumn{2}{c}{ Guanine ring } \\
$\mathrm{N}(1)$ & -0.02 \\
$\mathrm{C}(2)$ & 0.00 \\
${ }^{*} \mathrm{~N}(2)$ & -0.05 \\
$\mathrm{~N}(3)$ & 0.03 \\
$\mathrm{C}(4)$ & 0.05 \\
$\mathrm{C}(5)$ & 0.03 \\
$\mathrm{C}(6)$ & 0.00 \\
${ }^{*} \mathrm{O}(6)$ & 0.02 \\
$\mathrm{~N}(7)$ & -0.02 \\
$\mathrm{C}(8)$ & -0.05 \\
$\mathrm{~N}(9)$ & 0.01 \\
${ }^{*} \mathrm{C}\left(1^{\prime}\right)$ & 0.15
\end{tabular}

Table 5. Torsion angles $\left({ }^{\circ}\right)$

The torsion angle convention is that given by Sundaralingam (1969).

$\begin{array}{lr}\mathrm{C}(8)-\mathrm{N}(9)-\mathrm{C}\left(1^{\prime}\right)-\mathrm{O}\left(1^{\prime}\right) & 23 \cdot 8 \\ \mathrm{C}\left(4^{\prime}\right)-\mathrm{O}\left(1^{\prime}\right)-\mathrm{C}\left(1^{\prime}\right)-\mathrm{C}\left(2^{\prime}\right) & -28 \cdot 3 \\ \mathrm{O}\left(1^{\prime}\right)-\mathrm{C}\left(1^{\prime}\right)-\mathrm{C}\left(2^{\prime}\right)-\mathrm{C}\left(3^{\prime}\right) & 37.7 \\ \mathrm{C}\left(1^{\prime}\right)-\mathrm{C}\left(2^{\prime}\right)-\mathrm{C}\left(3^{\prime}\right)-\mathrm{C}\left(4^{\prime}\right) & -31 \cdot 3 \\ \mathrm{C}\left(2^{\prime}\right)-\mathrm{C}\left(3^{\prime}\right)-\mathrm{C}\left(4^{\prime}\right)-\mathrm{O}\left(1^{\prime}\right) & 16 \cdot 6 \\ \mathrm{C}\left(3^{\prime}\right)-\mathrm{C}\left(4^{\prime}\right)-\mathrm{O}\left(1^{\prime}\right)-\mathrm{C}(1) & 7 \cdot 2 \\ \mathrm{O}\left(3^{\prime}\right)-\mathrm{C}\left(3^{\prime}\right)-\mathrm{C}\left(4^{\prime}\right)-\mathrm{C}\left(5^{\prime}\right) & 145 \cdot 9 \\ \mathrm{C}\left(3^{\prime}\right)-\mathrm{C}\left(4^{\prime}\right)-\mathrm{C}\left(5^{\prime}\right)-\mathrm{O}\left(5^{\prime}\right) & 173.9 \\ \mathrm{O}\left(1^{\prime}\right)-\mathrm{C}\left(4^{\prime}\right)-\mathrm{C}\left(5^{\prime}\right)-\mathrm{O}\left(5^{\prime}\right) & 56 \cdot 2\end{array}$

of the $\mathrm{C}(6)-\mathrm{O}(6)$ bond from 1.228 to $1.38 \AA$. This partial loss of carbonyl character has affected the delocalization in the purine such that, for example, the $\mathrm{N}(1)-\mathrm{C}(6)$ bond has shortened from $1.402 \AA$ (Voet \& Rich, 1970) to $1.32 \AA$ and the angles $\mathrm{C}(5)-\mathrm{C}(6)-\mathrm{N}(1)$ and $\mathrm{C}(6)-\mathrm{N}(1)-\mathrm{C}(2)$ have changed from 111 and $125^{\circ}$ (Voet \& Rich, 1970), to 121 and $116^{\circ}$ respectively.

The ribose sugar shows a $\mathrm{C}\left(2^{\prime}\right)$-endo pucker [Fig. 2(b) and Table 4]. The phase angle of pseudorotation (Altona \& Sundaralingam, 1972), which expresses this puckering in a quantitative manner, has a value of $149.5^{\circ}$, which implies that the puckering is not far from being $\mathrm{C}\left(1^{\prime}\right)$-exo, as may be seen in Fig. 2(a). The glycosidic angle (Table 5), is in the usual anti region (e.g. Neidle, Taylor \& Robins, 1978). The exocyclic $\mathrm{C}\left(4^{\prime}\right)-\mathrm{C}\left(5^{\prime}\right)$ bond has a trans, gauche conformation (Table 5).

The crystal structure is devoid of hydrogen bonds (Fig. 3), the molecules being held together by van der Waals forces alone; there are no unusually close contact distances.

We are grateful to the Cancer Research Campaign and the Lawson Tait Trust for support, and Dr M. B. Hursthouse for access to his data collection facilities.

\section{References}

Altona, C. \& Sundaralingam, M. (1972). J. Am. Chem. Soc. 94, 8205-8212.

Bridson, P. K., Markiewicz, W. T. \& Reese, C. B. (1977). J. Chem. Soc. Chem. Commun. pp. 791-792.

Destro, R., Kistenmacher, T. J. \& Marsh, R. E. (1974). Acta Cryst. B30, 79-85.

De Titta, G. T., Edmonds, J. W., Langs, D. A. \& HauptMan, H. (1975). Acta Cryst. A31, 472-479.

KARLE, J. (1968). Acta Cryst. B24, 182-186.

MaIn, P. (1977). Acta Cryst. A33, 750-757.

Motherwell, W. D. S. (1975). Univ. of Cambridge. Unpublished.

Neidif,, S., TAylor, G. L. \& Robins, A. B. (1978). Acta Cryst. B34, 1838-1841.

Roberts, P. J., Pettersen, R. C., Sheldrick, G. M., IsaAcs, N. W. \& Kennard, O. (1973). J. Chem. Soc. Perkin Trans. 2, pp. 1978-1984.

SAEnger, W. (1973). Angew. Chem. Int. Ed. Engl. 12, 591601.

SHELdRICK, G. M. (1976). Direct Methods Symposium, Buffalo, New York. To be published.

Sundaralingam, M. (1969). Biopolymers, 7, 821-860.

Thewalt, U., Bugg, C. E. \& Marsh, R. E. (1970). Acta Cryst. B26, 1089-1101.

Voet, D. \& Rich, A. (1970). Prog. Nucleic Acid Res. Mol. Biol. 10, 183-265. 